\title{
Individual Differences in Auditory and Visual Attention Among Fetal Alcohol-Affected Adults
}

\author{
Paul D. Connor, Ann P. Streissguth, Paul D. Sampson, Fred L. Bookstein, and Helen M. Barr
}

\begin{abstract}
Background: Deficits in attention are commonly identified among patients who have been prenatally exposed to alcohol, and they often affect the ability of the patients to function appropriately in society.

Methods: Eleven adult patients with fetal alcohol syndrome (FAS) or fetal alcohol effects (FAE) were compared with nine adult subjects with no history of prenatal alcohol exposure, using four tests of visually and auditorially mediated attention.

Results: In relation to the comparison group, patients with FAS/FAE exhibited substantial deficits in both auditory and visual attention; the auditory deficits were greater. We observed two predominant patterns of deficits among patients with FAS/FAE, one involving both auditory and visual attention problems and the other involving less severe auditory problems and even fewer visual problems. Most subjects with FAS or FAE had some manifestations of attention problems in at least one of the tests of attention used in this study. We present a new graphical representation of individual auditory Continuous Performance Test data across a 6-min period, which, compared with conventional scores, more clearly reveals the markedly disrupted and variable attention patterns displayed by some individuals with FAS or FAE.

Conclusions: These findings suggest that detailed analysis of the pattern of individual performance for each subject is an important aspect of Continuous Performance Test assessment. Our findings further suggest that intellectual performance (intelligence quotient score) alone is not sufficient to account for the patterns of disrupted attention for individuals with FAS/FAE. Assessment of individuals with FAS or FAE should include measurement of attentional functioning in both the visual and auditory modalities.
\end{abstract}

Key Words: Visual Attention, Auditory Attention, Fetal Alcohol Syndrome, Individual Variability.

$\mathbf{A}^{17}$ TTENTION DISTURBANCES are a frequently cited area of concern for children and adolescents who have received diagnoses of fetal alcohol syndrome (FAS) or fetal alcohol effects (FAE). Steinhausen et al. (1982a) found that children of alcoholic mothers exhibited higher rates of concentration impairments than did either normal subjects or children of epileptic mothers. Similarly, in a recent study of patients with FAS/FAE, $60 \%$ of those 6 to 20 years of age were reported to have experienced attention problems, and $70 \%$ were reported to have repeatedly experienced difficulties paying attention at school (Streissguth and Kanter, 1997). Attention problems are one of the hallmarks of fetal alcohol behaviors, affecting many areas of the lives of the subjects, including work and school performance.

Mirsky et al. (1991) proposed a four-component theory

From the Departments of Psychiatry and Behavioral Sciences (P.D.C., A.P.S., H.M.B.) and Statistics (P.D.S.), University of Washington, Seattle, Washington, and the Institute of Gerontology (F.L.B.), University of Michigan, Ann Arbor, Michigan.

Received for publication November 5, 1998; accepted May 24, 1999.

This research was supported by Grant AA01455-01-21 from the National Institute on Alcohol Abuse and Alcoholism.

Reprint requests: Paul D. Connor, Ph.D., Department of Psychiatry and Behavioral Sciences, University of Washington, Fetal Alcohol and Drug Unit, 180 Nickerson Street, Suite 309, Seattle, WA 98109. Fax: 206-685-2903.

Copyright $\mathbb{0} 1999$ by the Research Society on Alcoholism. of attentional functioning to explain clinically observed attention disturbances. First is the ability to focus attention, i.e., to select one stimulus to attend to from among the other stimuli that are competing for attention. Deficits in this ability have been linked to damage to the right posterior cortex, especially the parietal cortex. Patients with FAS or FAE often become distracted by extraneous stimuli and have difficulty focusing their attention. The second element of attention proposed by Mirsky et al. (1991) is sustaining attention, i.e., the ability to maintain focus on a particular stimulus over time. Deficits in this ability have been linked to lesions of the frontal lobes, regions of the cingulate gyrus, thalamus, corpus striatum, and reticular formation. The third component of attention is the ability to flexibly shift attention between different stimuli, which is linked to the functioning of the anterior cingulate gyrus, prefrontal cortex, and medial frontal cortex. The fourth component of attention is encoding, i.e., the processing of numeric information. This has been linked to hippocampal functioning. Deficits in both the focusing and sustaining components of attention were significantly related to prenatal alcohol exposure in a birth cohort of 464 subjects participating in a population-based epidemiological study (Streissguth et al., 1994). Those studies of subjects with various levels of alcohol exposure showed that attention deficits were frequent symptoms of prenatal alcohol exposure from age 4 through 
14 years (Streissguth et al., 1984, 1986, 1993, 1994, 1995). The encoding component of attention described by Mirsky et al. (1991) was not addressed in the current small study. The components that were evaluated were focusing, shifting, and sustaining attention.

Nanson and Hiscock (1990) indicated that the performance of children with FAS/FAE in attention tasks was similar to that of children who had received diagnoses of attention deficit disorder. Both patient groups made significantly more impulsive errors in attention tasks than did a group of normal subjects. A similar pattern was found by Carmichael Olson et al. (1992) in a study of adolescents and adults. Patients with FAS were much more impulsive than the comparison group in tasks of letter cancellation and sustained visual attention. Unlike Nanson and Hiscock (1990), Coles et al. (1997) found that children with FAS performed somewhat differently in some tasks than did a group of children with attention deficit and hyperactivity disorder who showed similar intelligence quotient (IQ) scores. Children with attention deficit and hyperactivity disorder tended to have trouble focusing and sustaining attention, whereas children with FAS did not seem to have this difficulty as often. However, Coles et al. (1997) indicated that, because of the difficulty of the attention tasks that were administered, several practice trials were used. Subjects who were unable to complete the practice trials were not given the attention tasks. This would seemingly eliminate the low end of the distribution and bias the sample toward more highly functioning subjects. Therefore, these findings cannot be compared with the findings of other studies (Carmichael Olson et al., 1992; Nanson and Hiscock, 1990).

The majority of previous studies of attention among patients with FAS/FAE have focused on visual aspects. However, auditory attention is also important, as Mateer et al. (1990) described in their work on the rehabilitation of acquired attention deficits. Studies in other clinical populations, such as subjects with autism or schizophrenia, have found that auditory attention is often impaired, sometimes more than visual attention (Ciesielski et al., 1990; Grillon et al., 1991). To examine this in adolescents and adults with FAS, Kerns et al. (1997) performed a study with two tests of auditory attention. The 16 patients with FAS performed below normal levels on both auditory attention tasks (complex sustained attention and alternating attention to an auditory stimulus). Although their poorest performance was in verbal recall after a distraction task (counting backward), the subjects performed nearly perfectly under undistracted auditory recall conditions. This suggests that attention disturbances may not be noticed under simple conditions but may become evident with increasingly complex tasks.

To our knowledge, no investigation has assessed both visual and auditory attention, in the same study, with an FAS/FAE population. Also, most previous studies of alcohol-related attention problems focused on children and
Table 1. Demographic Subject Information

\begin{tabular}{lccc}
\hline & $\begin{array}{c}\text { FAS } \\
(n=5)\end{array}$ & $\begin{array}{c}\text { FAE } \\
(n=6)\end{array}$ & $\begin{array}{c}\text { Control } \\
(n=9)\end{array}$ \\
\hline Gender & & & \\
$\quad$ Male & 4 & 3 & 3 \\
$\quad$ Female & 1 & 3 & 6 \\
Age (years) & 20.4 & 21.2 & 20.8 \\
Education level & 11 & 12.2 & 13.4 \\
IQ score & 89 & 95 & $109^{\mathrm{a}}$ \\
Positive psychiatric history & $2 / 5$ & $4 / 6$ & $2 / 9$ \\
Learning disabilities/special & $3 / 5$ & $4 / 6$ & $2 / 9$ \\
$\quad$ education history & & & \\
History of substance abuse & $3 / 5$ & $0 / 6$ & $0 / 9$ \\
\hline
\end{tabular}

a The IQ difference between control subjects and patients with FAS was significant at the 0.05 level.

adolescents. The present study assesses young adults and measures both auditory and visual attention. We also present a new display method to help examine individual performance variability in a Continuous Performance Test (CPT) task.

\section{METHODS}

This report is derived from a pilot study for a large, 21-year, follow-up study assessing the neuropsychological sequelae of prenatal exposure to various alcohol levels. A sample of 11 nonretarded adult patients with FAS/FAE (summarized in Table 1) were selected from participants in the Fetal Alcohol Follow-Up Study of the University of Washington Fetal Alcohol and Drug Unit. All patients had been diagnosed as having FAS or FAE by experienced dysmorphologists, according to published criteria (Clarren and Smith, 1978). Patients ranged in age from 19 to 23 years. Education ranged from 9 to 13 years. IQ scores ranged from 75 to 117 . Patients with IQ scores within the mentally retarded range ( 70 and below) were excluded from this sample.

Subjects with FAS or FAE were compared with a group of nine nonpatients who were nonretarded, were matched for age, and had no known prenatal alcohol exposure, no history of head trauma involving loss of consciousness, no history of neurological disturbances, and no current psychiatric problems. The control group ranged in age from 19 to 24 years. Education ranged from 10 to 16 years. IQ scores ranged from 88 to 138 . Groups were not further matched with respect to IQ, because decreased IQ scores are frequently correlated with prenatal alcohol exposure.

Four tests of visual and auditory attentional functioning were administered as part of a larger neuropsychological battery of tests thought to be sensitive to the effects of prenatal alcohol exposure (Kerns et al., 1997; Streissguth et al., 1994). Two of these tests [the Letter Cancellation Test (LCT) and CPT] were selected from the National Institute of Mental Health Attention Battery (Mirsky et al., 1991) to measure focusing and sustaining attention, respectively. Two other tests were selected as auditory measures of attentional functioning [the Consonant Trigrams Task (CTT) and Attention Process Training Test (APT)]. The Wechsler Adult Intelligence Scale-Revised was used to assess the relationship between intelligence and attention. The full battery of tests averaged $6 \mathrm{hr}$ (range, 5-7.5 hr). The first of the attention tasks (LCT) was administered after approximately $2 \mathrm{hr}$ of testing and the second (CTT) after approximately 3 hr of testing. The last two (APT and finally CPT) ended the testing session. Additional tasks were not included because of the length of the battery. The four attention tests were administered as follows.

A shortened version of the Talland LCT (Talland, 1965), in which only one trial of each condition was used, was administered. This test measures the ability of the subjects to focus visual attention. The subjects are required to scan pages filled with letters, searching for either capital letters or double spaces on the page. False alarms and omissions were recorded for each search. 
Table 2. Group Means, SDs, and $t$ Ratios for 29 Attention Scores for Control Subjects and Patients with FAS/FAE

\begin{tabular}{|c|c|c|c|c|c|}
\hline \multirow[b]{2}{*}{ Tests } & \multicolumn{2}{|c|}{ FAS/FAE } & \multicolumn{2}{|c|}{ Control } & \multirow[b]{2}{*}{$t$ ratio ${ }^{a}$} \\
\hline & Mean & SD & Mean & SD & \\
\hline \multicolumn{6}{|l|}{ Visual battery } \\
\hline LCT capital letters omissions & 4.4 & 3.9 & 2.9 & 2.3 & +0.43 \\
\hline LCT capital letters false alarms & 0.8 & 1.3 & 0.3 & 0.7 & +1.05 \\
\hline LCT double space omissions & 4.2 & 4.6 & 2.1 & 2.9 & +1.39 \\
\hline LCT double space false alarms & 0.8 & 1.5 & 0.4 & 1.0 & +0.54 \\
\hline LCT both omissions & 5.1 & 6.4 & 2.7 & 3.0 & +0.72 \\
\hline LCT both false alarms & 0.6 & 1.8 & 0.2 & 0.7 & +0.51 \\
\hline CPT $X$ errors of omission & 1.5 & 2.2 & 0.1 & 0.3 & +1.97 \\
\hline CPT $X$ false alarms & 1.2 & 1.9 & 0.2 & 0.4 & +1.27 \\
\hline CPT $X$ reaction time & 40.6 & 4.1 & 37.4 & 3.0 & +1.87 \\
\hline CPT $X$ SD of reaction time & 8.3 & 1.9 & 8.0 & 2.2 & +0.36 \\
\hline CPT AX errors of omission & 4.8 & 4.6 & 2.1 & 3.1 & +1.63 \\
\hline CPT AX false alarms & 3.0 & 4.1 & 0.9 & 1.5 & +1.70 \\
\hline CPT AX reaction time & 9.4 & 4.4 & 7.9 & 3.1 & +0.14 \\
\hline CPT AX SD of reaction time & 35.3 & 7.3 & 35.8 & 9.4 & -0.86 \\
\hline \multicolumn{6}{|l|}{ Auditory battery } \\
\hline CPT tone errors of omission & 10.8 & 7.7 & 1.7 & 1.5 & +4.59 \\
\hline CPT tone false alarms & 19.6 & 37.4 & 1.6 & 1.1 & +1.98 \\
\hline CPT tone reaction time & 44.2 & 6.2 & 39.9 & 5.2 & +1.65 \\
\hline CPT tone SD of reaction time & 15.0 & 5.9 & 10.9 & 1.5 & +2.06 \\
\hline APT sustained attention correct responses & 27.5 & 2.6 & 29.9 & 0.3 & +2.99 \\
\hline APT sustained attention false alarms & 0.8 & 1.0 & 0.2 & 0.4 & +1.51 \\
\hline APT selective attention correct responses & 27.8 & 2.6 & 29.9 & 0.3 & +2.80 \\
\hline APT selective attention false alarms & 0.7 & 1.4 & 0.0 & 0.0 & +1.63 \\
\hline APT alternating attention correct responses & 18.1 & 7.4 & 25.8 & 5.9 & +2.09 \\
\hline APT alternating attention false alarms & 3.3 & 4.2 & 0.8 & 1.0 & +1.17 \\
\hline APT divided attention correct responses & 25.5 & 4.0 & 28.7 & 1.9 & +2.50 \\
\hline APT divided attention false alarms & 1.3 & 1.5 & 1.4 & 1.7 & -0.08 \\
\hline CTT 3-sec delay & 10.8 & 2.4 & 13.3 & 1.4 & +2.73 \\
\hline CTT 9-sec delay & 8.2 & 2.8 & 10.4 & 1.6 & +2.16 \\
\hline Cा 18-sec delay & 5.1 & 3.0 & 7.9 & 3.4 & +1.97 \\
\hline
\end{tabular}

a The $t$ ratios pertain to transformed variables (see text). The positive $t$ ratios are in the expected direction.

The Brown-Peterson CTT (Peterson and Peterson, 1959) requires the subjects to recall three orally presented consonants after 3-, 9-, and 18-sec distraction tasks (counting backward by threes). The CTT assesses the ability of the subjects to simultaneously maintain two sets of auditory information and to shift their attention between the two. The number of letters recalled after each delay period was recorded.

For the CPT (Sunrise Systems, Pembroke, MA) (Mirsky et al., 1991, 1992), three conditions were used, i.e., a simple item visual discrimination trial (X, 4 min in length), a trial in which the subjects were asked to respond to $\mathrm{X}$ only after presentation of $\mathrm{A}(\mathrm{AX}, 6 \mathrm{~min})$, and an auditory discrimination task ( $6 \mathrm{~min}$, responding to a high-pitched tone). Two of these tasks arc commonly used visual sustained attention tasks. The third task assesses an auditory version of sustained attention. Correct responses, omissions, and false alarms were tallied for each condition. Reaction times and SDs were computed.

The APT (Sohlberg and Mateer, 1989) is presented on audiocassette and assesses auditory attention processes under four conditions. (1) The sustained attention involves a task of simple stimulus discrimination, similar to the $X$ trial in the visual CPT. (2) The selective attention is similar to the AX condition in the visual CPT and involves another form of sustained attention ability in the model of attention described by Mirsky et al. (1991). (3) The alternating attention involves a task in which the subjects must alternate between response criteria (switching from responding to 2 followed by 5 to responding to 5 followed by 2), as an example of an auditorially mediated attention shift. (4) The divided attention requires the subjects to perform an auditory selective attention task while completing a visual scanning letter-cancellation task and is another test of shifting attention. Correct responses and false alarms were counted.

The four tests used in this study here generated 29 outcome scores. Three of these were counts of the number of correct responses (from the CTT), and six were reaction time means and SDs from the CPT. The remaining 20 were error scores. Because error scores typically have skewed distributions, these were transformed to logarithmic scores for analysis $[\log ($ number of errors +1$)]$. Group differences were assessed by $t$ tests of individual scores (Table 2), and first principal-component scores were computed separately for the auditory and visual tests (Fig. 1).

\section{RESULTS}

The results of the current study show that attention disturbances are seen in adults with FAS/FAE in both visual and auditory tests. In visual tasks of attention, all but one of the measures (SD of reaction time in the AX task of the CPT) showed differences in mean performance, in the expected direction of subjects with FAS/FAE performing more poorly than control subjects (Table 2 ).

Likewise, all of the tasks of auditory attention exhibited group mean differences in the expected direction, except for the number of false alarms in the APT divided attention task. In the CPT auditory task, patients with FAS/FAE made significantly more errors of omission and showed a higher SD of reaction time than did comparison subjects. They scored fewer correct responses under all four conditions of the auditorially based APT and fewer correct responses under all conditions of the CTT.

The first principal component of the 15 auditory scores included the auditory CPT, APT, and CTT. It was clearly defined, even in this small sample, as a fairly evenly 


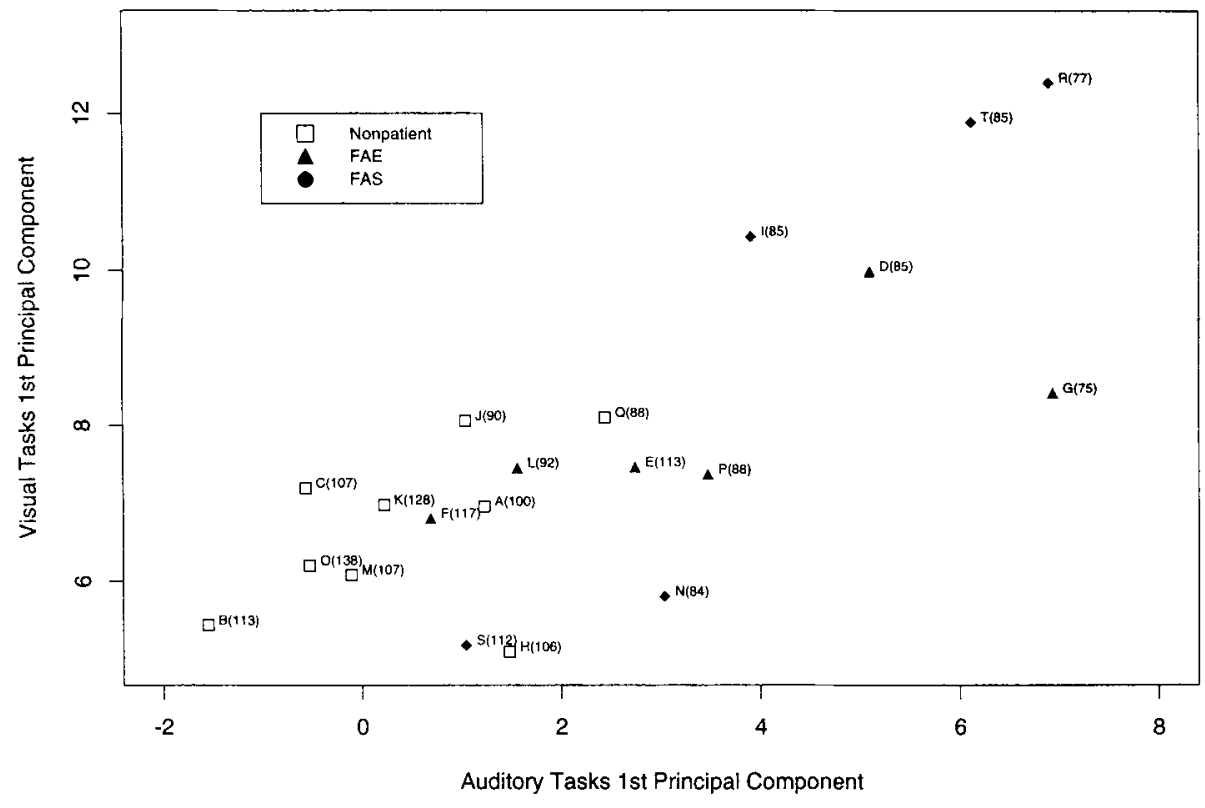

Fig. 1. Patterns of inattention. The net principalcomponent auditory attention scores are plotted against the net visual attention scores; points are labeled with the diagnoses and (in parentheses) the full-scale IQ scores. The scores on both axes represent a continuum of performance, in which a higher score on either axis indicates poorer performance on the principal component of attention. Letters identify single subjects. Within the FAS/FAE group, some subjects show deficits of both auditory and visual attention (upper right cluster) and others show neither (lower center, adjacent to control subjects). weighted combination of all of the (standardized) scores except for false alarms in the alternating attention and auditory divided attention tasks of the APT, which were weakly correlated with the rest of the battery. The 11 patients with FAS/FAE accounted for the eight worst (highest) scores in this auditory principal component. The first principal component of the 14 visual scores reflected mainly the visual CPT and LCT tasks. The five worst scores in this visual attention summary were observed for patients with FAS/FAE. These five subjects (Fig. 1, upper right cluster, subjects I, D, G, T, and R) clearly had difficulty with both visual and auditory tasks. Three additional patients with FAS/FAE had less severe auditory problems, and three exhibited results within the range of the control scores. All six of these latter patients showed results within the range of the control values for visual attention.

To test the significance of the group differences in the auditory and visual summary scores without making distributional assumptions of normality, we performed a permutation test (Good, 1994). The group labels "FAS/FAE" and "Control" were randomly permuted over the cases 1000 times, and the resulting two-sample $t$ statistics were computed to represent the "null" distribution corresponding to no group effect on the principal-component scores. The results (Fig. 1) indicate a clearly significant difference between patients and control subjects for the auditory tasks $(p=0.002)$ and a more modest difference for the visual tasks $(p=0.044)$.

The average difference indicated by these test statistics conceals more important patterns in Fig. 1. Figure 1 shows that the five patients with FAS/FAE and the poorest auditory and visual attention scores had five of the six lowest IQ scores. However, we also observed that the patients with FAS or FAE who had only moderate attention deficits showed a wide range of IQ scores (IQ scores of 84-113). In this small sample, IQ seemed insufficient to explain the differences in attention between these two groups of patients; larger studies will be needed to further evaluate this.

Some light was shed on the relationship between IQ and attention by examining the individual CPT tone records. Of the 29 attention scores represented in the principalcomponent analysis, the score for auditory tone errors of omission was the single most discriminating in separating subjects with FAS/FAE from control subjects ( $t$ ratio = +4.59 ) (Table 2). Compared with the use of only average scores for individuals as a measure of attention (as in Table 2 ), the patterns of successes and failures to attend across the entire $6 \mathrm{~min}$ of the auditory CPT provide more information regarding attention deficits. Figure 2 presents data for four subjects with similar IQ scores (subjects $N, J, T$, and D) who showed disparate temporal patterns of attention during the 6-min CPT tone task. Each graph in Fig. 2 depicts all responses made by one subject during this task.

The upper two graphs in Fig. 2 represent a control subject with an IQ of 90 (subject J) and a patient with FAS with an IQ of 84 (subject $N$ ). Their patterns of attention showed some similarities and some differences. They exhibited almost no false alarms or late responses and showed little variation in reaction time. However, the patient with FAS (subject $N$ ) made many errors of omission, primarily during the first half of the task, whereas the control subject did not. This patient with FAS (subject $\mathrm{N}$ ) apparently became attentive halfway through the task and then showed a performance record that was very similar to the record for the control subject. However, subject $\mathrm{N}$ accrued so many omission errors in the earlier portion of the test that his overall omission score $(n=13)$ was comparable to that of subject $\mathrm{D}(n=14)$ and worse than that of subject T $(n=$ 7), both patients showed markedly poor attention overall. The lower two graphs in Fig. 2 (subjects T and D) show 
Fig. 2. Individual differences in CPT tone task performance during a 6-min period. Runs of correct responses are represented by black lines. The tick marks on the horizontal axis represent the times at which the critical stimuli appeared. The tick marks on the vertical axis indicate the distribution of all reaction times. FSIQ, full-scale IQ score; VIQ, verbal IQ score; PIQ, performance IQ score.
Subject $\mathrm{J}:$ Nonpatient, $F S I Q=90 \mathrm{VIQ}=93 \mathrm{PIQ}=90$

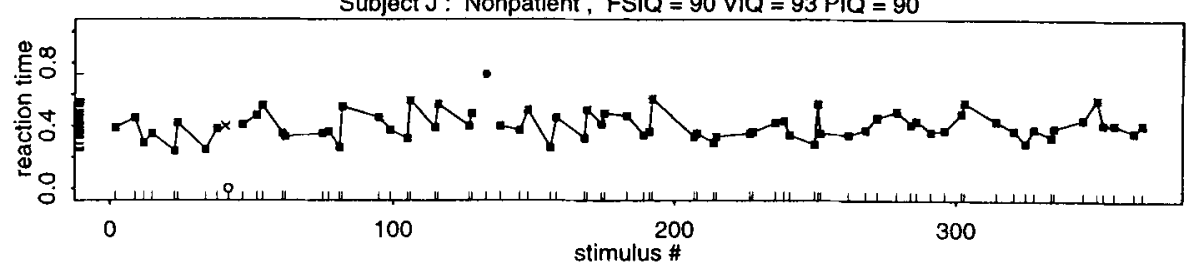

Subject $N$ : FAS, FSIQ $=84$ VIQ $=77 \mathrm{PIQ}=98$

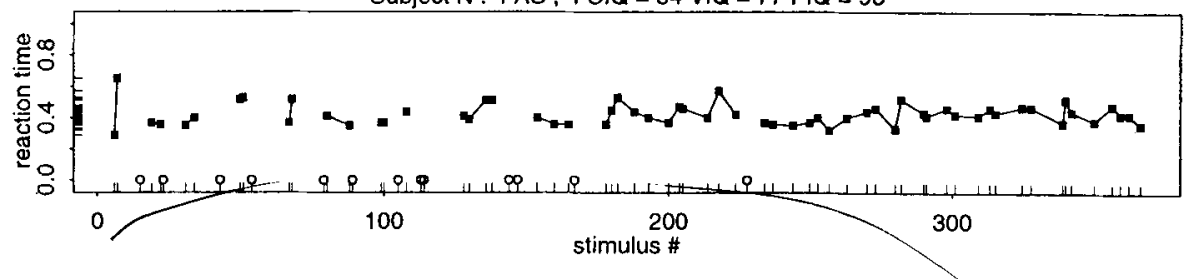

Subject $T:$ FAS, FSIQ $=85 \mathrm{VIQ}=79 \mathrm{PIQ}=98$

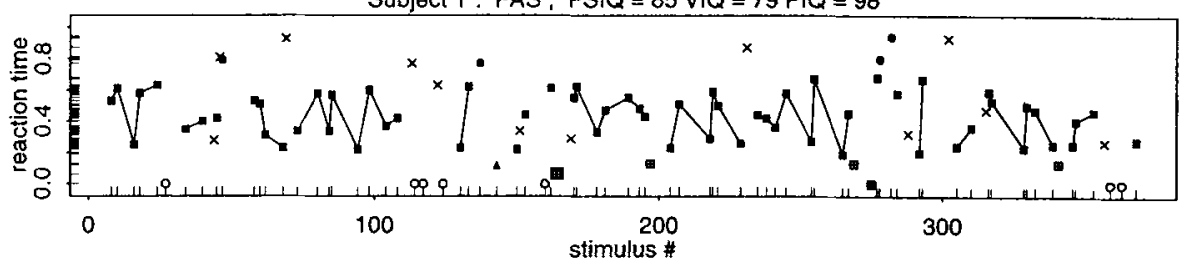

Subject $D: F A E, F S I Q=85$ VIQ $=87 P I Q=86$

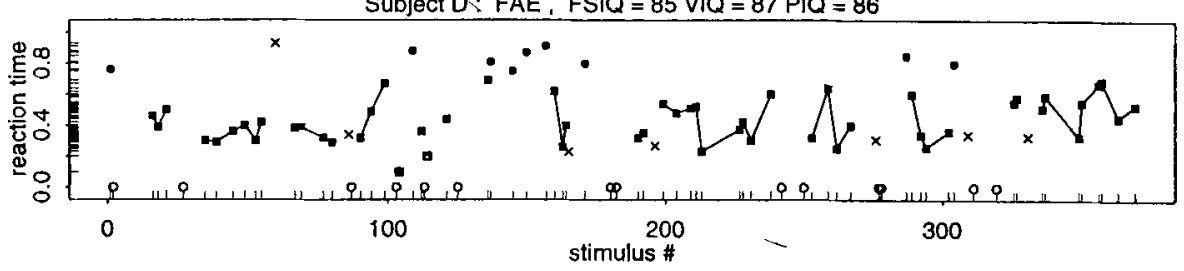

many errors of commission and omission, late responses spaced intermittently throughout the trial, only short runs of consecutive correct responses, and tremendous variability in reaction times. Subjects $T$ and $D$, who clearly have severely disrupted attention, are adults with FAS and FAE, respectively. Their performance was characteristic of that of four of the five patients in the subgroup in Fig. 1 (upper right cluster).

What accounts for this variability in attention? It does not seem to be a function of IQ alone. Subject $J$ (a nonpatient) exhibited a full-scale IQ of 90, whereas the two adults with FAS and the adult with FAE exhibited almost identical full-scale IQ scores $(84,85$, and 85 , respectively). Both subjects with FAS showed a 6- to 7-point discrepancy between verbal and performance IQ scores, with the verbal IQ score being lower. One might wonder whether some adverse environmental factors could account for these discrepant attention findings. However, all three of these adults with FAS or FAE were raised in nurturing families during childhood and had good educational opportunities, raising doubts regarding adverse environmental effects.

\section{DISCUSSION}

Several new findings are suggested by this small sample study. The presentation of individual performance records across time (Fig. 2) and the scatter comparing the performance of each individual in composites of both auditory and visual attention scores (Fig. 1) have facilitated detection of individual differences in attention that should be useful in understanding the functioning of alcohol-affected individuals.

Statistically significant attention deficits in both auditory and visual tests were observed for these patients with FAS/ FAE, but the strongest effects were observed in auditory tests. The three most effective tests in discriminating these 
deficits in patients with FAS/FAE were the CPT tone task, APT, and CTT (all three of the tests in the auditory battery). Not all scores for these tests were equally effective. The most discriminating scores were two of the four CPT tone task scores, four of the eight APT scores, and two of the three CTT scores. These eight most discriminating scores included seven that measured errors of omission and one (from the CPT tone task) that measured SD of reaction time.

One previous study of subjects with FAS or FAE found that $94 \%$ of the sample group reported histories of positive mental health problems (Streissguth et al., 1997). Table 1 indicates that six of our affected patients reported positive psychiatric histories, compared with only two control subjects. However, the worst performers in both auditory and visual attention tasks had not reported histories of psychiatric troubles. Seven affected patients, compared with two control subjects, reported histories of learning disabilities or participation in special education, a finding consistent with those of another study in this population (Streissguth et al., 1997). A history of learning disabilities does not seem to be a confounding variable, because attention can be considered a vital component of learning abilities.

In our study, patients with IQ scores within the mentally retarded range were excluded from the sample. In another study of 473 subjects with FAS or FAE, only $9 \%$ of the FAE sample and $27 \%$ of the FAS sample had IQ scores within the mentally retarded range (Streissguth and Kanter, 1997). Therefore, our decision to select patients with IQ scores of $>70$ did not severely truncate the range of IQ scores commonly observed for this population.

In the terminology of the theory of attention described by Mirsky et al. (1991), alcohol-affected subjects showed deficits in the focusing, sustaining, and shifting components of attention in the auditory modality and in the focusing and sustaining components of attention in the visual modality. Based on this, we could expect to find damage to many of the brain structures described by Mirsky et al. (1991) as being associated with these types of attention deficits. These include the parietal and frontal lobes, prefrontal and medial frontal cortex, cingulate gyrus, and reticular formation. Neuroimaging studies at various sites in the United States are underway to assess many of these brain regions.

This study suggests that the attention deficits so often noted in children and adolescents with FAS/FAE also occur in adulthood. However, the pattern of attention disturbances in these adults may be somewhat different from the familiar one. Whereas Carmichael Olson et al., (1992) and Nanson and Hiscock, (1990) found that children and adolescents with FAS exhibited many false alarms and were impulsive in their responses, the current study of adults with FAS indicates a somewhat different pattern. The adults with FAS or FAE in our sample made more errors of omission, in addition to errors of commission, and seemed somewhat inattentive, rather than solely impulsive. Previ- ous studies of children and adolescents showed attention disturbances in visual tasks, but auditory attention was not measured (Carmichael Olson et al., 1992; Coles et al., 1997; Nanson and Hiscock, 1990). The current study of adults with FAS/FAE reveals auditory attention deficits in addition to visual deficits. In that regard, our findings replicate those of the other study of adult patients with FAS using auditory attention tests, although that study did not measure visual attention (Kerns et al., 1997).

Auditory attention and visual attention have been jointly studied in a number of other populations. Patients with petit mal epilepsy who were evaluated with respect to event-related brain potentials exhibited auditory attention that was more impaired than visual attention (Duncan, 1988). In a CPT study of Irish schizophrenics and their relatives, Mirsky et al., (1995) found that auditory attention was much more impaired than visual attention; in fact, almost one-half of the schizophrenics and almost one-half of their schizophrenic adult relatives could not even complete the CPT auditory attention tasks, in contrast to nonschizophrenic relatives who had no trouble completing the tasks. A study of German schizophrenics also found auditory CPT performance more affected than visual CPT performance (Mussgay and Hertwig, 1990). In a more recent study of children with attention deficits, Mirsky et al., (in press) found similar discrepancies between auditory and visual attention in the CPT. However, in that group the discrepancy between the two modalities of attention was not as marked as in the schizophrenia or petit mal epilepsy studies. Further studies of auditory/visual discrepancies among subjects with FAS/FAE and other populations should be of interest.

In the present study, we detected a subgroup of five patients (some with FAS and others with FAE) who exhibited exceptionally large deficits in both modalities of attention. They shared another specific characteristic, namely high SDs of reaction times in the auditory task. Three of these patients also showed high SDs of reaction times in the AX task. In light of these findings (albeit from a small sample), we suspect that there is a heterogeneity hidden in these diagnoses; some patients with FAS/FAE have this distinct attention deficit and others do not. Although, on average, the patient group seemed to have more severe auditory deficits than visual deficits, the auditory and visual deficits were equally severe in this subgroup with greater deficits. Detection of this subgroup, which accounts for almost all of the mean differences between patients and the comparison group, required inspection of the scatter in Fig. 1 and was not apparent from statistical tests of group averages.

An important feature of the present study involves the greater clarity of individual deficits revealed by the individual 6-min performance records from the CPT auditory tone task. Attention lapses were revealed here that were not as discernible from average scores of individual performances across time. Here we see that marked differences in pat- 
terns of auditory attention were manifest even among individuals with similar IQ scores, from apparently similar benign environments. Further application of this methodology should be important in clinical settings.

Future studies should assess auditory attention in children and adolescents with FAS/FAE, to determine whether some have deficits similar to those found here. They should also explore the possible relationship between auditory attention and the auditory impairments known to be associated with prenatal alcohol exposure, by specifically testing for auditory impairments (Church and Kaltenbach, 1997; Rössig et al., 1994; Steinhausen et al., 1982b; Streissguth et al., 1985). The present study did not explicitly assess hearing acuity. Although no subject acknowledged a diagnosed hearing impairment or the use of hearing aids, the subjects could have been unaware of auditory problems, particularly if the problems had been lifelong. Visual disorders have also been noted for patients with FAS or FAE (Strömland and Hellström, 1996). Although this study did not specifically assess visual acuity or visual disorders, subjects wore corrective lenses when appropriate. Future studies would benefit from specific testing for visual disorders, to better explore possible relationships between these disorders and visual attention problems.

The current findings of auditorially mediated attention disturbances have important clinical implications. We think that auditory attention as well as visual attention should be assessed to more precisely characterize the deficits of patients with FAS/FAE. The attention disturbances observed for children with FAS/FAE do not disappear, as is often the case with facial malformations. The deficits are still present in adulthood, and they could negatively affect the ability of patients to function adequately and appropriately in employment environments and in social and personal relationships. In light of the considerable variability in performance among individuals, it is important that patients with FAS/ FAE be examined individually, with appropriate tests, to identify their particular patterns of cognitive strengths and weaknesses.

\section{ACKNOWLEDGMENT}

We gratefully acknowledge the consultation of Allan F. Mirsky, Ph.D. We also thank our psychometrist, Michael Hampton, who tested many of the people in this study, and our case manager, Kaylin Anderson, who found our patients and encouraged them to come in for testing. We are grateful to Bernard Weiss, Ph.D., who suggested examination of individual CPT performance.

\section{REFERENCES}

Carmichael Olson H, Fcldman J, Streissguth AP (1992) Neuropsychological deficits and life adjustment in adolescents and adults with fetal alcohol syndrome. Alcohol Clin Exp Res 16:380.

Church MW, Kaltenbach JA (1997) Hearing, speech, language, and vestibular disorders in the fetal alcohol syndrome: A literature review. Alcohol Clin Exp Res 21:495-512.
Ciesielski KT, Courchesne E, Elmasian R (1990) Effects of focused selective attention tasks on event-related potentials in autistic and normal individuals. Electroencephalogr Clin Neurophysiol 75:207-220.

Clarren SK, Smith DW (1978) The fetal alcohol syndrome. N Engl J Med 298:1063-1067.

Coles CD, Platzman KA, Raskind-Hood CL, Brown RT, Falek A, Smith IE (1997) A comparison of children affected by prenatal alcohol exposure and attention deficit, hyperactivity disorder. Alcohol Clin Exp Res 21:150-161.

Duncan C (1988) Application of event-related brain potentials to the analysis of interictal attention in the absence of epilepsy, in Elements of Petit Mal Epilepsy (Myslobodsky MS, Mirsky AF eds) pp 341-364, Peter Lang, New York.

Good PI (1994) Permutation Tests: A Practical Guide to Resampling Methods for Testing Hypotheses. Springer-Verlag, New York.

Grillon C, Ameli R, Courchesne E, Braff DL (1991) Effects of task relevance and attention on P3 in schizophrenic patients. Schizophr Res 4:11-21.

Kerns KA, Don A, Mateer CA, Streissguth AP (1997) Cognitive deficits in nonretarded adults with fetal alcohol syndrome. J Learn Disabil 30:685-693.

Mateer C, Sohlberg MM, Youngman PK (1990) The management of acquired attention and memory deficits following mild closed head injury, in Cognitive Rehabilitation in Perspective (Wood RL. Fussey I eds) pp 68-95, Lawrence Erlbaum Associates, London.

Mirsky AF, Anthony BJ, Duncan CC, Ahearn MB, Kellam SG (1991) Analysis of the elements of attention: A neuropsychological approach. Neuropsychol Rev 2:109-145.

Mirsky AF, Lochhead SJ, Jones BP, Kugelmass S, Walsh D, Kendler KS (1992) On familial factors in the attentional deficit in schizophrenia: A review and report of two new subject samples. J Psychiatr Res 26:383-403.

Mirsky AF, Pascualvaca DM, Duncan CC, French LM (1999) A model of attention and its relation to ADHD. Ment Retard Dev Disabil Res Rev, in press.

Mirsky AF, Yardlcy SJ, Jones BP, Walsh D, Kendler KS (1995) Analysis of the attention deficit in schizophrenia: A study of patients and their relatives in Ireland. J Psychiatr Res 29:23-42.

Mussgay L, Hertwig R (1990) Signal detection indices in schizophrenics on a visual, auditory, and bimodal Continuous Performance Test. Schizophr Res 3:303-310.

Nanson JL, Hiscock M (1990) Attention deficits in children exposed to alcohol prenatally. Alcohol Clin Exp Res 14:656-661.

Peterson LR, Peterson MJ (1959) Short-term retention of individual verbal items. J Exp Psychol 58:193-198.

Rössig C, Wässer S, Oppermann P (1994) Audiologic manifestations in fetal alcohol syndrome assessed by brainstem auditory-evoked potentials. Neuropediatrics 25:245-249.

Sohlberg MM, Mateer CA (1989) Introduction to Cognitive Rehabilitation: Theory and Practice. The Guilford Press, New York.

Steinhausen HC, Nestler V, Huth H (1982a) Psychopathology and mental functions in the offspring of alcoholic and epileptic mothers. J Am Acad Child Adolesc Psychiatry 21:268-273.

Steinhausen HC, Nestler V, Spohr HL (1982b) Development and psychopathology of children with fetal alcohol syndrome. J Dev Behav Pediatr 3:49-54.

Streissguth AP, Barr H, Kogan J, Bookstein F (1997) Primary and secondary disabilities in fetal alcohol syndrome, in The Challenge of Fetal Alcohol Syndrome: Overcoming Secondary Disabilities. University of Washington Press, Seattle.

Streissguth AP, Barr HM, Sampson PD, Parrish-Johnson JC, Kirchner GL, Martin DC (1986) Attention, distraction and reaction time at age 7 years and prenatal alcohol exposure. Neurobehav Toxicol Teratol 8:717-725.

Streissguth AP, Bookstein FL, Sampson PD, Barr HM (1993) The Enduring Effects of Prenatal Alcohol Exposure on Child Development: Birth Through 7 Years, a Partial Least Squares Solution. University of Michigan Press, Ann Arbor, MI. 
Streissguth AP, Bookstein FL, Sampson PD, Barr HM (1995) Attention: Prenatal alcohol and continuities of vigilance and attentional problems from 4 through 14 years. Dev Psychopathol 7:419-446.

Streissguth AP, Clarren SK, Jones KL (1985) Natural history of the fetal alcohol syndrome: A ten-year follow-up of eleven patients. Lancet 2:85-91.

Streissguth AP, Martin DC, Barr HM, Sandman BM, Kirchner GL, Darby BL (1984) Intrauterine alcohol and nicotine exposure: Attention and reaction time in 4-year-old children. Dev Psychol 20:533-541.
Streissguth AP, Sampson PD, Carmichael Olson H, Bookstein FL, Barr HM, Scott M, Feldman J, Mirsky AF (1994) Maternal drinking during pregnancy and attention/memory performance in 14-year-old children: A longitudinal prospective study. Alcohol Clin Exp Res $18: 202-218$

Strömland K, Hellström A (1996) Fetal alcohol syndrome: An ophthalmologic and socioeducational prospective study. Pediatrics 97:845850.

Talland GA (1965) Deranged Memory. Academic Press, New York. 